\title{
An Appropriate Solution of Designing and Accomplishing of a Geodetic Network for Monitoring of the Constructions
}

\author{
Constantin COSARCA ${ }^{1 *}$, Aurel SARACIN ${ }^{1}$, Aurel Florentin NEGRILA ${ }^{1}$, Adrian SAVU ${ }^{1}$ \\ ${ }^{1}$ Department of Topography and Cadastre, Faculty of Geodesy, Technical University of Civil Engineering \\ Bucharest, Romania. \\ *)corresponding author, e-mail: constantin_cosarca@yahoo.com
}

BulletinUASVM Horticulture 72(2) / 2015

Print ISSN 1843-5254, Electronic ISSN 1843-5394

DOI:10.15835/buasvmcn-hort:11370

\begin{abstract}
The monitoring of the behaviour of spatial structure of a building is a systematic activity of collecting and capitalizing of information derived from observation and measurements of some phenomena and sizes that characterizes the structure in the process of interaction with the environment and technological developments. Verifications by calculating the amount "stress values" in the characteristic sections of the building are confronted with the results of tests on models or test sections. These are - currently - followed by observations made by means of measures installed both in the body of the building and outside it. Hypothesis verification models are supplied by statistical tests. On the measurements compensation there are formulated several hypotheses. To validate or not the results obtained after compensation is necessary to verify such assumptions. To detect possible deformations (displacements) occurring between two networks, observed at different times, the analysis should be performed only on the remaining common (identical) points in the interval $\mathrm{T}_{\mathrm{i}}$ and $\mathrm{T}_{\mathrm{i}+1}$. In principle, comparing the coordinates (i.e. altitudes / heights) of the network points determined at different stages and investigate whether they form or not congruent figures. The difference between the determined parameters for network points should be within the "safety margin". The safety margin is calculated according to the empirical standard deviation. If this does not fall within safe limits, the statistic test does not indicate anything other than that deformations have appeared in the network.
\end{abstract}

Keywords: accurate measurements, compensation, displacements, stability, statistical tests.

\section{INTRODUCTION}

The current development in construction design and execution, which is becoming wider, more complex and more precise, requires an exigent control (Cosarca et al., 2008). This is possible to achieve - among others - by methods of Engineering Geodetic Measurements, discipline that is based on the geodetic measurement methods (Onose et al., 2006). The determination of the accurate spatial positions of the characteristic points of structural elements of the building, through the specific measurement methods and if possible - in a short time, has an important role in this area (Onose et al., 2007).
In principle, in Romania, a technical documentation is based on the provisions of the no.10/1995 Law, regarding the construction quality. The general principles for in time monitoring of constructions behaviour are stipulated by the Normative P1301999 which corresponds to the provisions of the 10/1995 Law regarding the construction quality and of the Regulation regarding the behaviour monitoring in exploitation, timely interventions and post-use of constructions approved by Government Decision no. 766/1997.

Monitoring of the behaviour of the spatial structure of a building is a systematic activity of collecting and capitalizing the information 
derived from observations and measurements of some phenomena and sizes that characterizes the structure in the process of interaction with the environment and technological developments (Romania. Ministry of Public Works and Regional Planning (1999). Standard on the tracking of the behavior in time of construction P130-1999).

This article presents an extremely important aspect on the obligation to achieve a geodetic reference network able to ensure - through its own points - a stable reference in all measurement stages provided of the monitoring program. The answer to this question is given - among others - by application of modern analysis methods, which involve the application of statistical tests on the stages of measurement and from which results conclusions about the stability of geodetic reference network points. The paper has an original character and is a synthesis of scientific research concerns of a restrains collective from Faculty of Geodesy of the Technical University of Civil Engineering Bucharest.

\section{MATERIALS AND METHODS \\ Methodsfor determining the displacements and deformations of constructions}

There are several criteria after which the classifications of research and observation methods shallbe made. Thus the classifications may be dependent on the deformation type, equipment type and where the equipment is located during the research (Onose, 2004). From a practical point of view, an appropriate classification can be made depending on the place of the equipment during research. Thus, the methods are divided into (Onose, 2004):

- Physical methods (measuring equipment fit directly onto the studied construction, or inside it);

- Geometric methods (determines the position of the control points relative to the fixed network points located outside the area of influence of the construction).

By means of these methods it can be determined the absolute sizes of displacements and deformations of construction. The geometric methods, that can be geodetic or photogrammetric, are used in the final phase of exploitation and tracking time of the behaviour of constructions. We can notice that the geodetic measurements can be extended to all exposed areas to displacements or deformations.

Given the proposed topics to be approached in this article, namely monitoring the horizontal and vertical displacements of the construction of the Glina Wastewater Treatment Plant, the following methods are proposed to be used distinctly or combined (Onose et al., 2009a,b):

- High precision geometric levelling;

- High precision trigonometric levelling;

- Linear and angular measurements of high precision.

For the geodetic reference network points it is considered that at each cycle of measurements the stability analysis of these points has to be done. If the points will be considered unstable, they

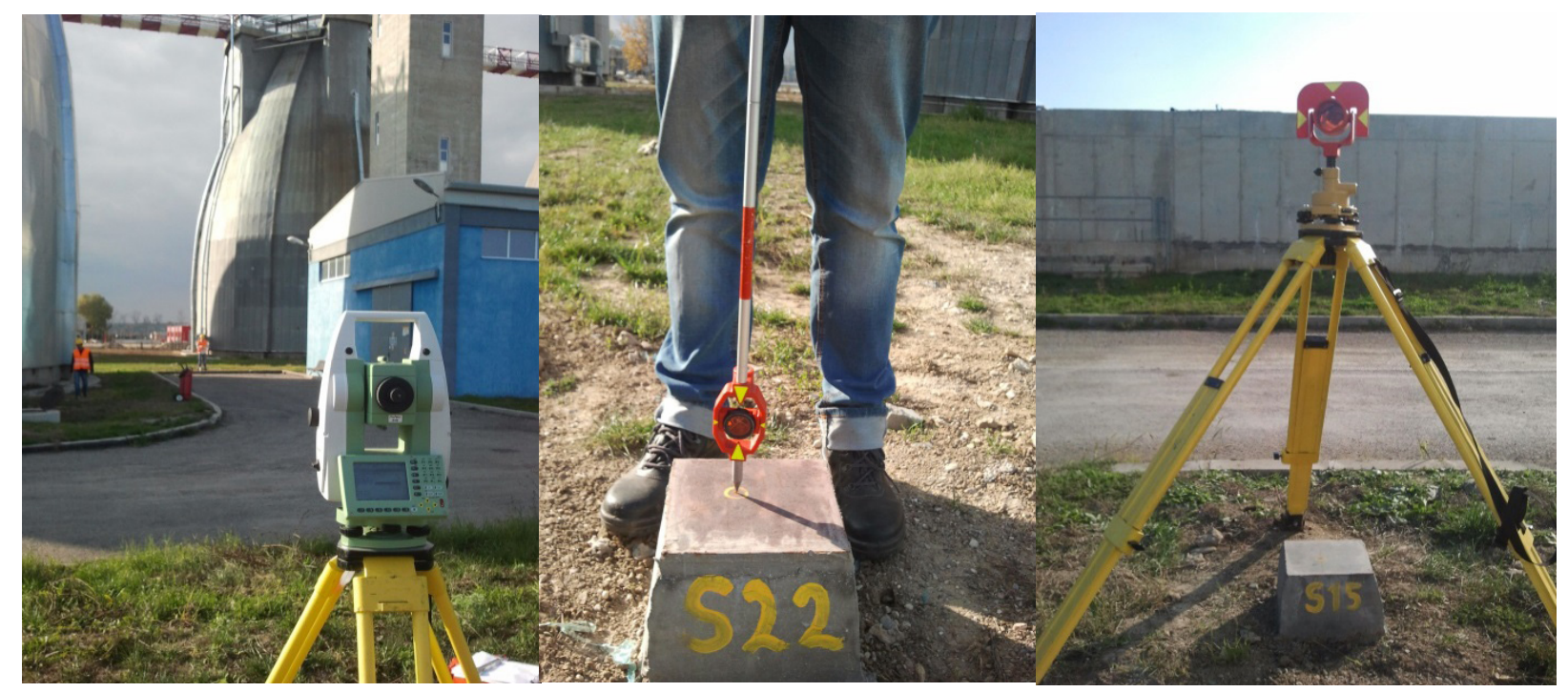

Fig. 1. Aspects during the measurements 
will still be considered and monitored as "object points".

\section{The geodesic reference network}

To demonstrate the theoretical notions presented in the research work were used the measurements performed in a reference combined Geodetic Network, a network of monitoring in time of an important engineering construction, namely the Wastewater Treatment Station Glina, Bucharest.

According to the project, the reference monitoring system is assured by a geodesic network consisting of 23 points materialized using concrete pillars located around the park SFT (Sludge Fermentation Tanks) (Sărăcin et al., 2013).

In this article has been started from the premise that all measurements are performed with conventional means of measuring respectively high performance total stations and levelling instruments (Fig. 1).

Using a high performance total station (Leica TCR 1201) and their reflective prisms (Leica type) the angular measurements were performed (horizontal and zenith angles) and also, the distances in almost all the possible combinations between the base points S1, S2, ... S23 which constitute the planimetric reference for observing the positions of the sight targets from the monitored objectives (Fig. 2).

From the most convenient station points were also targeted points on the SFT (Sludge Fermentation Tanks) which represent the object

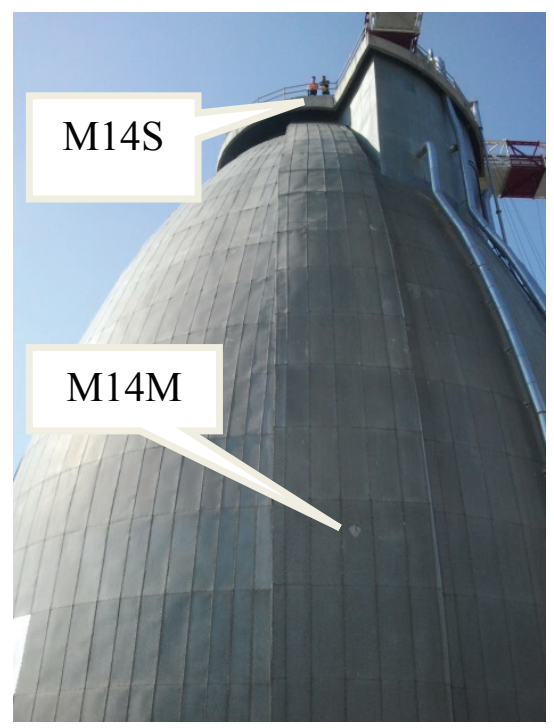

points, subject to tracking from three levels, in order to determine their spatial position (Fig. 3).

\section{points}

The determination of stability of control

The classic method of verifying the stability of the control points (landmarks of the geodetic reference network) involves the comparison of the values of the coordinates, respectively level differences between landmarks obtained from „initial” measurements compared to the „current” measurements.

The modern methods involve using statistical tests on the datas from different stages of measurement from which we will have the conclusions about the stability of the landmarks.

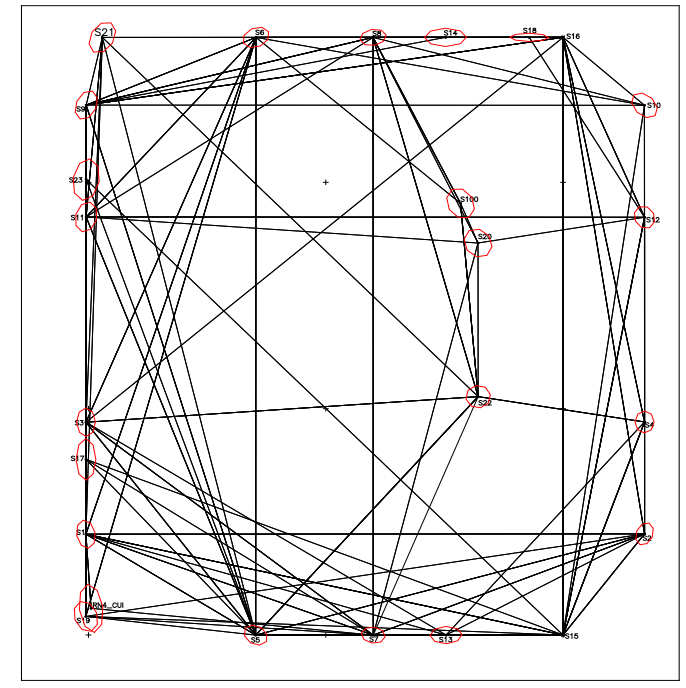

Fig. 2. Sketch of the geodetic network

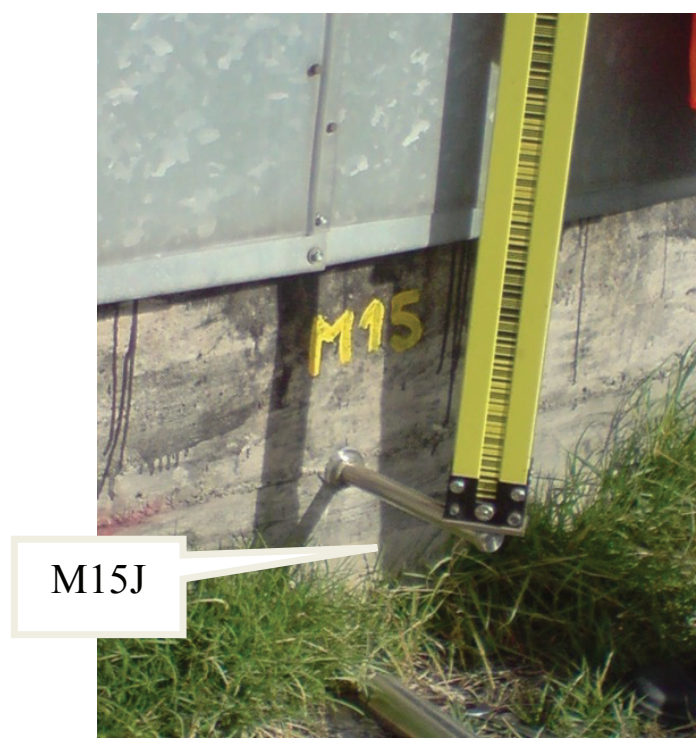

Fig. 3. Points materialized on the monitored objective 
If the resulted coordinates differ, the level measured between the initial stage and the current stages (measurements carried out between the reference marks) does not differ by more than the measurement error then the landmarks can be considered stable.

Classical Mathematical models used to determine the points displacements

In specialty literature there are presented 4 models (Onose, 2004):

\section{The static model}

This model is the most common. During performing observations it is considered that there are no deformations. When using this model, we do not consider the causes that provoked the deformations. This model starts from the idea that there were no deformations and the monitoring geodetic network has the same configuration in all stages of measurement. Based on these considerations we shall define in statistical terms the "zero hypothesis":

$$
\left(\mathbf{H}_{0}\right): \mathbf{E}\left(\mathbf{X}_{1}\right) \equiv \mathbf{E}\left(\mathbf{X}_{2}\right) \equiv \ldots \equiv \mathbf{E}\left(\mathbf{X}_{\mathbf{n}}\right)
$$

Where: $\mathbf{E}(\mathbf{X})$ - the expected value of the parameter $\mathbf{X}$ (mathematical expectation);

Within such a model it is tested if the "zero hypothesis" is true.

\section{If: $\mathbf{E}\left(\mathbf{X}_{1}\right) \equiv \mathbf{E}\left(\mathbf{X}_{2}\right) \rightarrow\left(\mathbf{H}_{0}\right)$ true}

$\mathbf{E}\left(\mathbf{X}_{1}\right) \neq \mathbf{E}\left(\mathbf{X}_{2}\right) \rightarrow\left(\mathbf{H}_{0}\right)$ shall be null (false); in its place it appears another hypothesis (H1) alternative hypothesis, true.

\section{The quasi-static model}

This model is derived from static models, it keeps the same concepts, but the monitoring of the geodetic networks is treated on two levels. In this case, we start using the new concepts of control point and of object point. The "zero hypothesis" is formulated just for the control points.

\section{The kinematic model}

This model aims to determine the deformations considering the time factor, but do not take into account the generating causes of the phenomenon of deformation. The behaviour in time of the points is deduced by statistical methods, allowing the determination of the speed and acceleration, being able to make interpolations, extrapolations and predictions.

\section{The dynamic model}

This model has in view the establishment of displacements in time taking into account the generating causes, taking into account the forces that cause displacements. The dynamic model describes using mathematical relations, the connection between cause and effect.

Here are two types of frequently used models:

a) - stochastic models; in this case measurement must be performed on the causes and the transmission functions are established on a statistical basis and based on the determination of some parameters. In this way there may be determined the displacements at certain measurement stages;

b) - deterministic models; in this situation the forces generating the phenomenon of displacement are known, using the differential equations and the finite element method to determine the displacements at certain measurement stages;

The deformation analysis between the two stages of measurement (Onose, 2004)

The models previously presented allowed only a comparison between two cycles of measurements. Currently, these mathematical models have been developed and allow the processing of several phases.

\section{The global test of congruence}

In principle, we compare the point coordinates of the geodetic network measured at different stages. It is necessary - in principle - to draw conclusions whether these points form or not congruent figures (Fig.4).

The difference between the network points parameters determined in two phases must be within a "safety limit", which is determined based on the empirical standard deviation of measurement (Fig.5).

If the results aren't in "safety limit", the comparison indicates that in the network we have deformations.

The functional model (Wolf et al., 1997)

$$
\underline{\mathbf{L}}_{\mathbf{i}}+\underline{\mathbf{V}}_{\mathbf{i}}=\underline{\mathbf{A}}_{\mathbf{i}} \cdot \underline{\hat{\mathbf{X}}}_{\mathbf{i}}
$$

where:

$$
\underline{\hat{\mathbf{X}}}_{\mathbf{i}} \text { - the parameters vector; }
$$

$\underline{\mathbf{A}}_{\mathbf{i}}$ - the configuration matrix; 

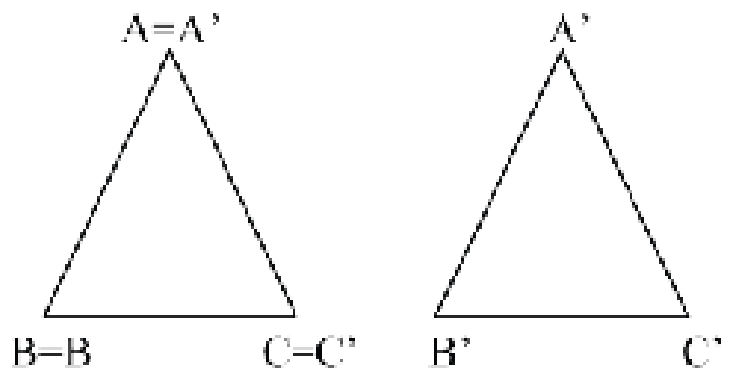

Fig. 4. Figures with equal elements

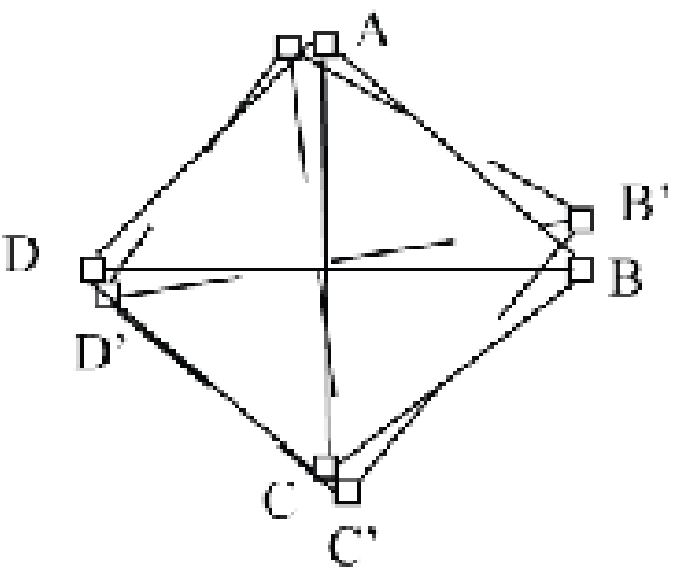

Fig. 5. Geodetic Network points in two stages

$\underline{\mathbf{V}}_{\mathbf{i}}$ - the corrections vector;

$\underline{\mathbf{L}}_{\mathbf{i}}$ - the free terms vector (the measurements vector);

\section{The stochastic model:}

$$
\begin{aligned}
& \underline{\Sigma} \mathbf{L}_{\mathbf{i}}=\sigma_{0}^{2} \cdot \mathbf{Q}_{\mathbf{L}} \\
& \mathbf{Q}_{\mathbf{L}}^{-1}=\mathbf{P}_{\mathbf{i}}
\end{aligned}
$$

where:

$\mathbf{Q}$ - the cofactors matrix;

$\sigma_{0}$ - the standard deviation;

$\mathbf{P}$ - the weights matrix.

The functional model (2) is subjected to the minimum condition:

$$
\underline{\mathbf{V}}^{\mathbf{T}} \underline{\mathbf{P}} \underline{\mathbf{V}}=\mathbf{m i n}
$$

In order to determine the displacements we could make a joint processing of both measurement stages:

$$
\left[\begin{array}{c}
\underline{\mathbf{L}}_{1} \\
\hdashline \underline{\mathbf{L}}_{2}
\end{array}\right]+\left[\begin{array}{l}
\underline{\mathbf{V}}_{1} \\
\hdashline \underline{\mathbf{V}}_{2}
\end{array}\right]=\left[\begin{array}{c:c}
\underline{\mathbf{A}}_{1} & 0 \\
\hdashline 0 & \underline{\mathbf{A}}_{2}
\end{array}\right] \cdot\left[\begin{array}{l}
\hat{\hat{\mathbf{X}}}_{1} \\
\underline{\hat{\mathbf{X}}}_{2}
\end{array}\right]
$$

The conditions for that the test of global congruence to locate the displacements in the studied network (Onose, 2010):

a. - in both stages of measurements it should be used the same provisional coordinates, in order to refer to the same DATUM;

b. - for both stages of measurement we must have the same DATUM defect;

c. - for determining the deformations, there are not used constrained networks, but unconstrained networks treated as free networks;

d. - the network configuration in both measurement stages should be the same;

e. - the theoretical global standard deviation $\sigma_{0}^{(1)}$ and $\sigma_{0}{ }^{(2)}$ must be the same for both measurement stages:

$\sigma_{0}^{(1)}=\sigma_{0}^{(2)}=\sigma_{0}$

After compensation it can be tested whether this condition is met by comparing the empirical standard deviation obtained after compensation using the Fisher statistical test, whose statistical practice is determined by the relationship (Pelzer, 1971):

$$
F_{\text {practical }}=\frac{\left(s_{0}^{(1)}\right)^{2}}{\left(s_{0}^{(2)}\right)^{2}}
$$

is compared with $\mathbf{F}_{\text {Theoretic }}=\mathbf{F}_{\mathrm{t} 1, \mathrm{t} 2, \boldsymbol{\alpha}}$ where:

$\mathbf{t}_{1}$ - number of degrees of freedom that allowed the determination of $\mathrm{s}_{0}{ }^{(1)}$;

$\mathbf{t}_{2}$ - number of degrees of freedom that allowed the determination of $\mathrm{s}_{0}{ }^{(2)}$;

$\boldsymbol{\alpha}$ - the confidence coefficient (the safety threshold).

If after the testing, the global theoretical standard deviation appears the same for both measurement stages, the global congruence test 
is made for the two configurations of the stages $t_{1}$ and $t_{2}$.

Out of the compensation we shall use the following elements:

- the measurements stage $t_{0}$ :

$$
\hat{\mathbf{X}}_{1} \quad \mathbf{Q}_{\mathbf{x} 1}=\mathbf{N}_{1}^{+}
$$

- the measurements stage $t_{1}$ :

$$
\hat{\mathbf{X}}_{2} \quad \mathbf{Q}_{\mathbf{x}} 2=\mathbf{N}_{2}^{+} \quad \mathbf{s}_{Q}
$$

where:

$$
\begin{aligned}
& \hat{\mathbf{X}}_{1}, \hat{\mathbf{X}}_{2}=\text { the parameters vector at the } \\
& \text { respective stage; }
\end{aligned}
$$

$\mathbf{Q}_{\mathbf{x} 1}, \mathbf{Q}_{\mathbf{x} 2}=$ the cofactors matrix at the respective stage;

$\mathbf{s}_{\mathbf{0}}, \mathbf{s}_{\mathbf{Q}} \quad=$ the empirical standard deviation at the respective stage;

With this data will be calculated:

$\emptyset \mathbf{d}=$ the discrepancies vector:

$$
\mathbf{d}=\hat{\mathbf{X}}_{2}-\hat{\mathbf{X}}_{1}
$$

$\emptyset \mathbf{Q}_{\mathrm{dd}}=$ the cofactors matrix of the deformation model:

$$
\begin{aligned}
& \mathbf{Q}_{\mathbf{d}}=\mathbf{Q}_{\mathbf{x} 1}+\mathbf{Q}_{\mathbf{x} 2} \\
& \mathbf{Q}_{\mathbf{d}}=\mathbf{N}_{1}^{+}+\mathbf{N}_{2}^{+} \\
& \mathbf{Q}_{\mathbf{d}} \Rightarrow \mathbf{Q}_{\mathbf{d}}^{+}
\end{aligned}
$$

$\emptyset \mathbf{s}_{0}=$ the empirical standard deviation of the deformation model:

$$
\mathbf{s}_{0}= \pm \sqrt{\mathbf{s}_{0}^{2}+\mathbf{s}_{\square}^{2}}
$$

$$
\emptyset \mathbf{h}=\text { the matrix rank } \mathbf{Q}_{\mathbf{d}}^{+}
$$

$$
\mathbf{h}=\mathbf{n}-\mathbf{d}
$$

Ø $\mathbf{F}=$ the calculated value of the Fischer test:

$$
\mathbf{F}=\frac{\mathbf{d}^{\mathbf{T}} \cdot \mathbf{Q}_{\mathbf{d}}^{+} \cdot \mathbf{d}}{\mathbf{h} \cdot \mathbf{s}_{0}^{2}}
$$

$\mathbf{F}_{\text {lim }}=$ the theoretical value of the Fischer test is extracted according to $\mathbf{a}, \mathbf{h}, \mathbf{f}$

$\mathbf{a}=$ the confidence coefficient (the safety threshold); $\alpha=95 \%$ $\mathbf{h}=$ the rank of the cofactors matrix of the deformation model

$\mathbf{f}=$ the number of degrees of freedom of the deformation model

- If: $\quad \mathbf{F} \leq \mathrm{F}_{\text {lim }} \Rightarrow\left(\mathrm{H}_{0}\right)$ true $\Rightarrow$

we do not have displacements in the network

$$
\mathbf{F}>\mathrm{F}_{\text {lim }} \Rightarrow\left(\mathrm{H}_{0}\right) \text { false } \Rightarrow
$$

we have displacements in the network

The global test of congruence indicates the fact that in the analyzed time frame, displacements occurred without indicating in which of the points.

For localization, there are several testing possibilities, the most common being the Student test (" $\mathbf{t}$ "). This test checks each parameter considered in the discrepancy vector, if it falls within defined safety limits.

It is calculated for each point:

$$
\begin{aligned}
& \emptyset \underline{\mathbf{d}}_{\mathbf{j}}=\underline{\mathbf{x}}_{2 \mathbf{j}}-\underline{\mathbf{x}}_{1 \mathbf{j}} \\
& \emptyset \mathbf{s}_{\mathbf{j}}=\mathbf{s}_{0} \sqrt{\mathbf{Q}_{\mathbf{j}}}
\end{aligned}
$$

under the root square, we can find the elements of the main diagonal of the cofactors matrix of the deformation model;

With the aid of the above elements we can determine the practical value of statistics ' $\mathbf{t}$ ' for the point $\mathbf{j}$ :

$$
\mathbf{t}_{\mathbf{j}}=\frac{\mathbf{d}_{\mathbf{j}}}{\mathbf{s}_{\mathbf{j}}}
$$

At this test it shall be considered as fix, the points that have the individual empirical standard deviation smaller than the empirical standard deviation of the deformation model:

$\mathbf{s}_{\mathrm{j}} \leq \mathbf{s}_{\mathbf{0}}$ - do not make research of deformations.

Because the all sizes ",n” are not independent stochastically, from statistical point of view calculated should be another risk factor $\bar{\alpha}$ resulting by solving the binomial equation:

$(1-\bar{\alpha})^{\mathbf{n}}=1-\alpha \Rightarrow \bar{\alpha}$ 
From statistical point of view $\bar{\alpha}$ could be estimated

$$
\text { as } \bar{\alpha}=\frac{\alpha}{\mathbf{n}}
$$

$\mathbf{t}_{\lim }=$ the theoretical value of the Student test is extracted depending on $\bar{\alpha}$ and $\mathbf{f}$;

If: $\quad \mathbf{t}_{\mathrm{j}} \leq \mathbf{t}_{\lim } \quad$ - the hypothesis $\left(\mathbf{H}_{\mathbf{0}}\right)$ true, the point $j$ is considered fixed;

$\mathbf{t}_{\mathbf{j}}>\mathbf{t}_{\lim } \quad$ - the hypothesis $\left(\mathbf{H}_{\mathbf{0}}\right)$ false, the point $\mathrm{j}$ is considered moved.

This test gives good results if the number of moved points is far smaller than the number of fixed points.

\section{RESULTS AND DISCUSSION}

In the above mentioned geodetic network were carried out successive measurements of geometric precision levelling, distances, horizontal directions, vertical angles in order to determine the vectors of movement for the levelling / sight marks mounted on the structural elements of the building in exploitation.

For the study case, there were considered a number of 3 stages of measurements made on the 23 reference points, located outside the zone of influence of the construction.

The processing of the measurements was performed using specialized software components, tested in numerous other similar works. To apply statistical tests there were performed and used specific software components.

Analyzing the planimetric geodetic network between stages $\mathbf{0}$ and $\mathbf{6}$ and $\mathbf{0}$ and $\mathbf{1 5}$ we can draw the following conclusions regarding the displaced points (see Tab.1 and Tab.2):

Between stages 0 and 6 we have 6 moved points because of the works that were done in the area of point S22 and for the other points because of the settlement of concrete pillars.

Between stages $\mathbf{0}$ and $\mathbf{1 5}$ the number of moved points increased also because of the works that were done in the area and for point S10 we have the bigest value because it was destroyed and replaced.

After the analysis between two stages, the points that have been identified as displaced were considered further that "object point" or "new
Tab. 1. The comparison between stages $\mathbf{0}$ and $\mathbf{6}$ we have 6 moved points:

\begin{tabular}{ccc}
\hline Point & $\begin{array}{c}\text { 'd }{ }_{\mathrm{j}} \mathrm{m} \text { ) } \\
\text { (the discrepancies } \\
\text { values) }\end{array}$ & $\begin{array}{c}\mathrm{s}_{\mathrm{j}}(\mathrm{m}) \\
\text { (standard } \\
\text { deviation of the } \\
\text { discrepancies) }\end{array}$ \\
\hline $\mathrm{S} 1$ & 0.003 & 0.001 \\
\hline $\mathrm{S} 12$ & 0.005 & 0.001 \\
\hline $\mathrm{S} 15$ & 0.005 & 0.001 \\
\hline $\mathrm{S} 18$ & 0.007 & 0.001 \\
\hline $\mathrm{S} 22$ & 0.010 & 0.001 \\
\hline S100 & 0.006 & 0.001 \\
\hline
\end{tabular}

Tab. 2. The comparison between stages $\mathbf{0}$ and $\mathbf{1 5}$ we have 12 moved points:

\begin{tabular}{ccc}
\hline Point & $\begin{array}{c}\text { 'd } \mathrm{d}_{\mathrm{j}}(\mathrm{m}) \\
\text { (the discrepancies } \\
\text { values) }\end{array}$ & $\begin{array}{c}\mathrm{s}_{\mathrm{j}}(\mathrm{m}) \\
\text { standard } \\
\text { deviation of the } \\
\text { discrepancies) }\end{array}$ \\
\hline $\mathrm{S} 1$ & 0.003 & 0.001 \\
\hline $\mathrm{S} 3$ & 0.009 & 0.001 \\
\hline $\mathrm{S} 4$ & 0.005 & 0.001 \\
\hline $\mathrm{S} 5$ & 0.009 & 0.001 \\
\hline $\mathrm{S} 7$ & 0.117 & 0.001 \\
\hline $\mathrm{S} 10$ & 22.483 & 0.001 \\
\hline $\mathrm{S} 12$ & 0.025 & 0.001 \\
\hline $\mathrm{S} 15$ & 0.006 & 0.001 \\
\hline $\mathrm{S} 20$ & 0.018 & 0.001 \\
\hline $\mathrm{S} 21$ & 0.005 & 0.001 \\
\hline $\mathrm{S} 22$ & 0.057 & 0.001 \\
\hline $\mathrm{S} 100$ & 0.070 & 0.002 \\
\hline
\end{tabular}

points" in compensation of the geodesic reference network.

In the case of in time monitoring of constructions the purpose is to determine if the building or the materialized points of respective building were moved or not.

If they were displaced the following questions appearing:

Where exactly was found / registered the displacement?

The answer to this question is suggested in content of this research work.

The accuracy of the measurements is the most important criteria. The choice of the geodetic method used in the monitoring is done according 
the nature of the monitored phenomenon. The choice of methods and measuring instruments shall be in accordance with the order of size of deformation. The processing of measurements is necessary to be performed using specialized software components, tested in works of this kind, to ensure the certainty of the results quality. The application of statistical tests is mandatory to determine the stability of control landmarks. This operation ensures the certainty that the possible displacements of object points are real and certain, meaning that the displacements values are independent of accuracy of measurements.

Represents or not this displacement a danger for the respective construction works?

The answer to this question is given after a deep analysis performed by an interdisciplinary team that participate in fulfilling the desideratum of this activity.

\section{CONCLUSION}

The monitoring activity of the behaviour of constructions is mandatory for all those involved (investors, designers, executants, owners, administrators, users, experts, specialists, responsible for monitoring the behaviour of constructions).

The quality of the constructions is the result of the totality of their behaviour performance in exploitation in order to satisfy the requirements of users and communities for the entire duration of their existence. Constructions behaviour monitoring is a component of quality system in construction.

The details of the current or special monitoring - period, methods, features and parameters followed - are established by the designer or expert, depending on the category of importance of constructions and other their characteristics. All these are included in the Technical Book of the construction that will include also the recorded results of these activities.

\section{REFERENCES}

1. Cosarca C, Onose D, Savu A, Negrilă A (2008). Geometrical Expertise and Dimensional Control of the Elements of Construction to the Hydrotechnics Buildings. Journal of Geodesy and Cadastre, RevCAD 8:9-16.

2. Onose D (2004). Constructions Behaviour Monitoring. Lecture Notes, Faculty of Geodesy, Technical University of Civil Engineering Bucharest.

3. Onose D, Savu A, Negrilă A (2005). The current stage in tracking deformations the bridge over Danube-Black Sea channel at Cernavoda. Journal of Geodesy, Cartography and Cadastre 14:206-214.

4. Onose D, Savu A, Negrilă A (2006). The determination of deformations in tracking geodetic networks. Journal of Cadastre, RevCAD 6:169-176.

5. Onose D, Cosarca C, Savu A, Negrilă A (2007). The geometric expertise of current state of lock Portile de Fier 1 through high precision measurements. Scientific Bulletin of Technical University of Civil Engineering Bucharest 3:3-15.

6. Onose D, Savu A, Negrilă A (2009a). Tracking behaviour in time of the bridge over the Danube - Black Sea channel from Cernavoda. Proceedings of the 11th WSEAS International Conference on Sustainability in Science Engineering 9:207-212.

7. Onose D, Cosarca C, Savu A, Negrilă A (2009b). Special networks used for tracking metal parts of the sluice. Proceedings of the 11th WSEAS International Conference on Sustainability in Science Engineering 9:213-217.

8. Onose D (2010). 3D Monitoring of Land and Buildings. Lecture Notes, Faculty of Geodesy, Technical University of Civil Engineering Bucharest.

9. Pelzer H (1971). Zur Analyse geodätischer Deformationsmessungen. Technischen Universitat Carolo Wilhelmina München.

10. Romania. Ministry of Public Works and Regional Planning (1999). Standard on the tracking of the behavior in time of construction P130-1999.

11. Sărăcin A, Cosarcă C, Savu A, Negrilă A (2013). Analysis of displacements and deformations for a Sludge Fermentation Tank (SFT4) of the Wastewater Treatment Plant Glina. Journal of Geodesy and Cadastre, RevCAD 15:261-270.

12. Wolf PR, Ghiliani C D (1997). Adjustment Computations: Statistics and Least Squares in Surveying and GIS. Published by Wiley-Interscience, 1997. 